\title{
Analysis of Factors Associated With Length of Stay of Opioid-Related Emergency Department Visits
}

Keshab Subedi 1

1. iREACH, ChristianaCare Health Systems, Wilmington, USA

Corresponding author: Keshab Subedi, keshab9subedi@gmail.com

\begin{abstract}
Introduction and Objective: Emergency department (ED) length of stay (LOS) is an important indicator of the quality of care in ED and is associated with patients' outcomes and healthcare costs. However, there is limited data on how the patient characteristics affect the ED LOS of opioid-related visits. This study aims to identify and quantify the effect of patient-related characteristics on LOS of opioid-related ED visits.
\end{abstract}

Methods: This is a retrospective analysis of electronic health records (EHR) of patients with diagnoses of opioid abuse. The study included patients with a diagnosis of opioid abuse who visited the ED at Christiana Care Hospital from January 1, 2017, to December 31, 2018 (N=5,661). The opioid-related visits were identified using ICD-10 diagnosis codes. We used accelerated failure time (AFT) models, a time-to-event analysis approach to evaluate the relationships of different patient characteristics with ED LOS.

Results: The mean age of the study population was 39 years. The study population had $40 \%$ female, $20 \%$ Black/African American, and 5\% Hispanic or Latino. The prevalence of co-use of cocaine and co-use of alcohol was $11 \%$, and $9 \%$, respectively. Also, $58 \%$ had mental health comorbidity, and $1 \%$ were homeless. The distribution of ED LOS was right-skewed with a median of 4.3 (IQR: 2.6, 6.8). Co-use of alcohol (time ratio, TR: 1.31 , CI: 1.23-1.40), co-use of cocaine (TR: 1.18 , CI: 1.11-1.25), the presence of mental health comorbidity (TR: 1.05, CI 1.01-1.09), and homelessness (TR: 1.57 , CI: 1.32-1.86) were associated with increased ED LOS.

Conclusions: Co-use of alcohol, co-use of cocaine, homelessness, and mental health comorbidity are associated with the longer LOS of opioid-related ED visits.

Review began 06/21/2021 Review ended 06/29/2021 Published 07/06/2021

\section{๑) Copyright 2021}

Subedi. This is an open access article distributed under the terms of the Creative Commons Attribution License CC-BY 4.0., which permits unrestricted use, distribution, and reproduction in any medium, provided the original author and source are credited.
Categories: Emergency Medicine, Internal Medicine, Epidemiology/Public Health

Keywords: emergency department, length of stay, opioid abuse, substance use disorder (sud), accelerated failure time model

\section{Introduction}

Delays in emergency department (ED) patient disposition lead to poor patient outcomes and increased healthcare costs. ED length of stay (LOS) has been used as one of the indicators of quality of care in the ED [1] and is reported to be both the cause and result of ED crowding forming a vicious cycle [2]. Health policymakers and healthcare providers have instituted a variety of metrics, such as door-to-diagnostic time, door to treatment time, and ED arrival to ED departure time to evaluate and improve the quality-of-care delivery in the ED [3]. Due to the opioid epidemic in the United States, a substantial proportion of ED visits are opioid-related. The rate of opioid-related ED visits increased from 87 per 100,000 population in 2008 to 242 per 100,000 population in 2016 [4]. Further, substance abuse has been attributed to increased resource utilization and longer ED LOS [5-7]. Therefore, it is important to understand the determinants of the LOS of opioid-related visits, especially given that the ED boarding, the practice of waiting in the ED for transition to an inpatient setting or to another facility, has become another pervasive public health problem [8]. Numerable studies have explored the factors associated with ED LOS, focusing both on the entire ED population [9-11], and specific patient's population such as surgical critical care patients [12,13], psychiatric and mental health patients [14-17], and ethanol intoxicated patients [18]. However, there is limited data on how the patient characteristics affect the LOS of opioid-related ED visits. We hypothesize that certain patient characteristics like co-use of alcohol, co-use of cocaine, presence of mental health comorbidity, and homelessness might increase LOS of opioid-related ED visits. This study aims to describe patients with opioid-related visits in ED of a Level 1 trauma center and to identify and quantify the effect of patientrelated characteristics on LOS of opioid-related ED visits. A better understanding of the determinants of LOS of opioid-related ED visits could help identify patient populations who require special intervention or resources. These findings could also help prevent protracted ED stay while improving both the quality of care and patients' outcomes.

\section{Materials And Methods}




\section{Study design and setting}

This is a retrospective analysis of electronic health records (EHR) of the patients with a diagnosis of opioid abuse who visited the ED at Christiana Care Hospital from January 1, 2017, to December 31, 2018. Christiana Care Health System is one of the largest healthcare providers in the mid-Atlantic with a Level 1 trauma center serving all of Delaware and parts of Pennsylvania, Maryland, and New Jersey. This study was approved by the Christiana Care Health System's institutional review board.

\section{Data and data preparation}

The analysis dataset was extracted from the EHR system of the Christiana Care Health System. Opioidrelated visits were identified using ICD-10 diagnosis codes: F11.x, R78.1, and T40.0x to T40.6x [19]. This definition encompasses both prescription and nonprescription abuse of opioids, including heroin, carfentanil, fentanyl, and methadone19. This study included only those visits that ended with direct discharge from ED. The ED visits in which the patient died, was kept in 23-hour observation, admitted to an inpatient or another facility were excluded from the analysis. The visits in which patients left against medical advice were also excluded from the analysis.

The demographic information in the data included patients' age, sex, race, and ethnicity. We used the Elixhauser algorithm [20] based on ICD-10 codes to identify the major comorbidities including AIDS, diabetes, hypertension, liver disease, hypothyroidism, neurological disease, pulmonary disease, anemia deficiency, arthritis, blood loss, and coronary artery disease. Other variables included the patients' mode of arrival, patients' primary insurance type, triage severity index, and homelessness. The mode of ED arrival was regrouped in to two groups - "ambulance arrival" if the mode of arrival was coded as ambulance arrival, and "other" if the mode of arrival was other than ambulance arrival including missing. The ED arrival shift was computed based on ED registration time. We also identified if the patient was co-using other substances, such as alcohol, and cocaine during the visit, in addition to opioid using ICD-10 diagnosis codes [21]. The LOS was defined and computed as the time from registration in the ED to discharge from the ED.

\section{Statistical analysis}

Summary statistics were calculated for patient's demographic and clinical variables. For categorical variables proportions were calculated, and for numerical variables mean and standard deviation are reported. We defined discharge from the ED as an event of interest and followed time-to-event analysis approaches. We plotted the Kaplan-Meir curve of survival (still being in the ED) stratified by patients' age category, sex, race, ethnicity, insurance type, the presence of mental health comorbidity, co-use of cocaine, co-use of alcohol, and homelessness. The differences in the Kaplan-Meir curves were tested using the logrank test. The multivariable accelerated failure time (AFT) models were used to evaluate the effect of patients' demographic and clinical characteristics on the LOS of ED visits. The AFT model is a type of survival analysis that directly models the log of time to an event as a function of a vector of model covariates [22]. We fitted the AFT models with exponential, log-normal, gamma, and Weibull distribution. The best fit model was selected using a likelihood-ratio test, and Akaike information criteria (AIC). The variables included in the models were guided by the clinical relevance rather than preliminary univariate analysis or arbitrary threshold P-value. The independent variables included in the models were age, sex, race, ethnicity, insurance type (private/ Medicare/Medicaid/ no insurance), the presence of mental health comorbidity (yes/no), arrival shift (morning/day/evening), weekend arrival (yes/no), co-use of alcohol (yes/no), co-use of cocaine (yes/no), and homelessness (yes/no). For ease of interpretation, the estimates from the AFT model are presented as time ratio (TR) calculated as e $\beta$, where $\beta$ is the regression coefficient from the AFT model. The TR, also referred to as an acceleration factor, can be interpreted as a ratio of LOS of the patients at a given level of a categorical variable to the patients at the reference level of the categorical variable. For numerical variables, the TR is the ratio of LOS corresponding to one unit change in the numerical variable.

Three percent of the visits had at least one of the variables of interest missing. The visits with a missing value(s) were removed from analysis using a pairwise deletion approach resulting in an available case analysis, where cases were excluded from only operations in which data were missing on a variable that was required. Statistical analyses were done using SAS $9.4^{\circledR}$ (SAS Institute, Cary, NC, USA) and the graphs were plotted using R.

\section{Results \\ Patient characteristics}

We identified $5661 \mathrm{ED}$ visits with a diagnosis of opioid abuse during the study period. The mean age of the patients was 39 years (SD:12.77) where $40 \%$ of the patients were women, $23 \%$ were Black/African American, and $5 \%$ were Hispanic or Latino. The majority of the patients (57\%) had Medicaid coverage, followed by private insurance (15\%), and Medicare (10\%), while $12 \%$ had no insurance coverage. The majority were classified as level 2 (44\%) and level 3 (42\%) in the triage severity index (Table 1). The most prevalent comorbidities were depression (52\%) followed by alcohol use disorder (39\%), chronic pulmonary disease (36\%), and psychosis (35\%). 


\section{Cureus}

\section{Variable}

Age [mean (SD)]

Sex: Female

Race: Black/African American

Ethnicity: Hispanic or Latino

Insurance: Medicaid

Insurance: Medicare

Insurance: Private

No Insurance Coverage

ED arrival mode: Ambulance

Weekend Arrival

Arrival Shift: Day

Arrival Shift: Evening

Arrival Shift: Night

Triage Severity Index: 1

Triage Severity Index: 2

Triage Severity Index: 3

Triage Severity Index: 4

Triage Severity Index: 5

Co-use of Alcohol

Co-use of Cocaine

Mental Health Comorbidity

Homeless
Total patients $(\mathrm{N}=5,661)$

39 (12.77)

2,246 (39.67)

$1,305(23.05)$

262 (4.63)

3,527 (56.84)

$602(10.63)$

832 (14.70)

700 (12.37)

2,899 (51.21)

$1,532(27.08)$

2,225 (39.30)

2,378 (42.01)

1,058 (18.69)

$64(1.23)$

2,470 (43.86)

2,403 (42.45)

628 (11.09)

96 (1.70)

492 (8.69)

898 (10.56)

$3,310(58.47)$

$65(1.15)$

TABLE 1: Characteristics of the study population. The values are count and percentage unless otherwise noted.

ED - emergency department

\section{Results from univariate analysis of the ED LOS}

The distribution of ED LOS was right-skewed with a median of 4.3 hours (IQR: 2.6, 6.9) (Figure 1). The probability of still being in the ED after one hour, three hours, and six hours were $95 \%, 68 \%$, and $32 \%$ respectively. The Kaplan-Meir curves of the probability of still being in ED stratified by age category, sex, race, ethnicity, co-use of alcohol, co-use of cocaine, the presence of mental health comorbidity, and homelessness are presented in Figures 2, 3. The survival plots plotting the probability of still being in ED were significantly different for the strata of age category $(\mathrm{p}<0.001)$, race $(\mathrm{p}<0.001)$, ethnicity $(\mathrm{p}=0.007)$, couse of alcohol $(p<0.001)$, co-use of cocaine $(p<0.001)$, the presence of mental health comorbidity $(p<0.001)$, and homelessness $(\mathrm{p}<0.001)$ with older patients, Black/African American, Hispanic or Latino, co-use of alcohol, co-use of cocaine, the presence of mental health comorbidity, and homeless having prognosis of longer ED LOS (Figures 2, 3). The median LOS of visits with and without co-use of alcohol was 6.1 vs 4.2 hours. Similarly, the median LOS was 5.5 vs 4.2 hours for visits with and without co-use of cocaine, 4.7 vs 3.9 hours for visits with and without mental health comorbidity, and 8.3 vs 5.3 hours for visits with and without homelessness. The median LOS for visits with co-use of both alcohol, and cocaine was 8.4 hours. 


\section{Cureus}

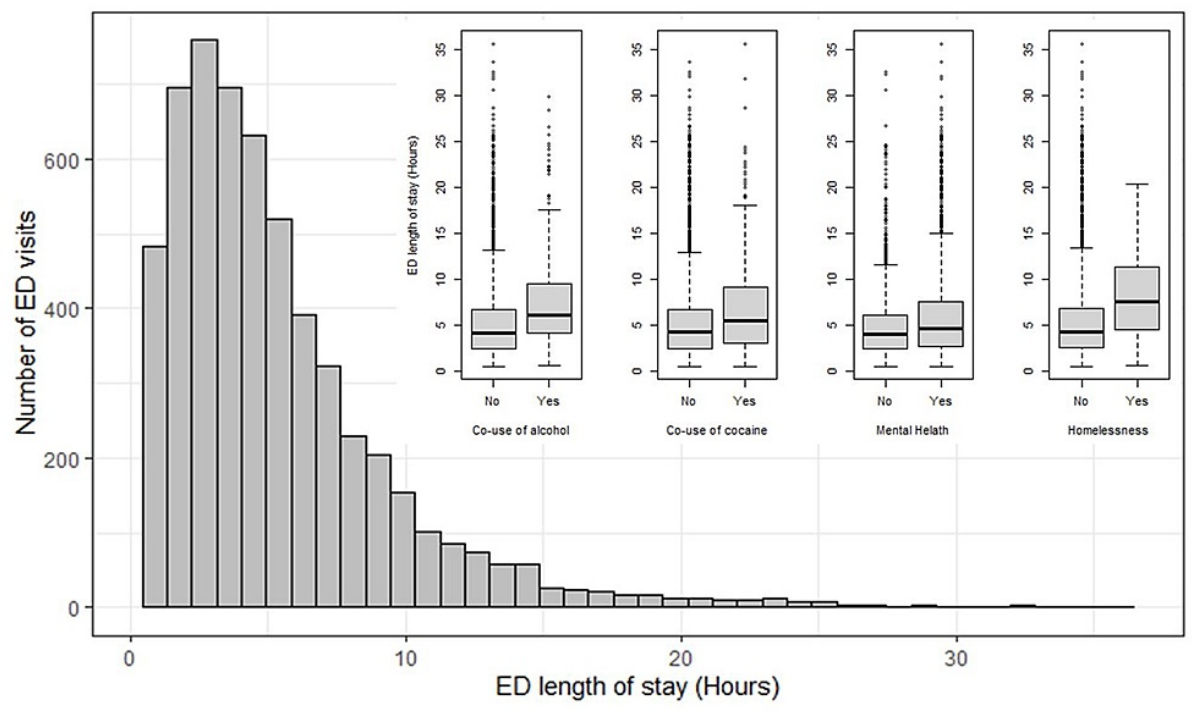

FIGURE 1: Distribution of the length of stay of opioid-related ED visits.

The box plots show the comparisons of ED length of stay of the patients with and without co-use of alcohol, co-use of cocaine, mental health comorbidity, and homelessness (from left to right).

ED - emergency department
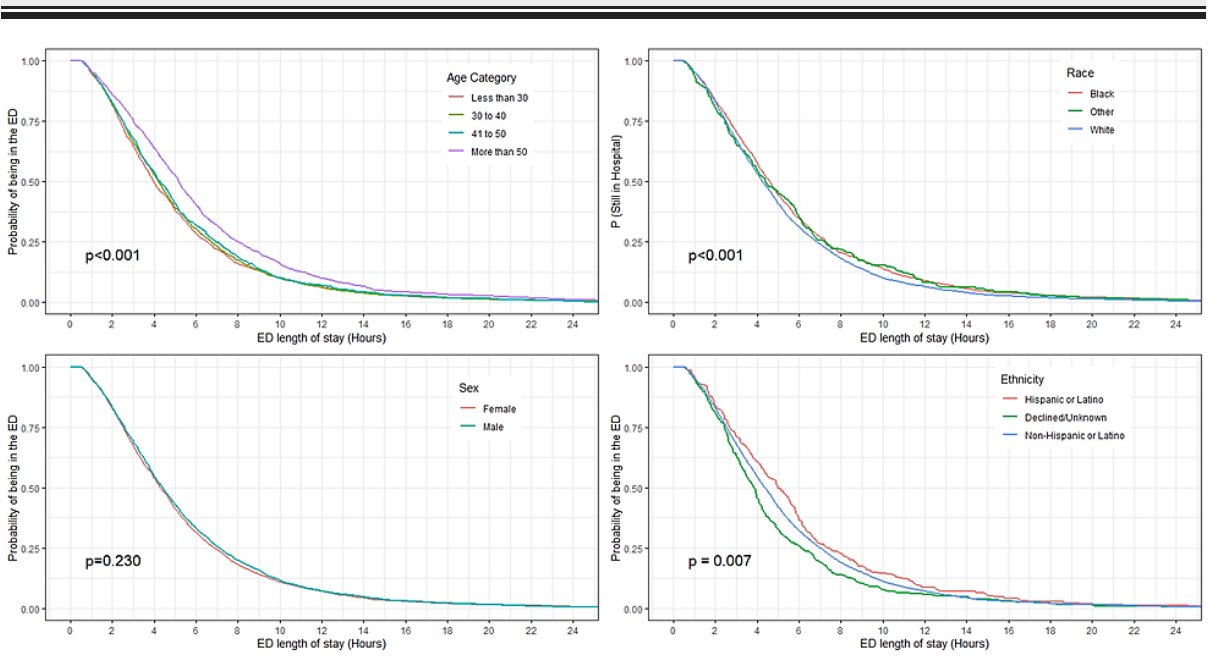

FIGURE 2: Kaplan-Meir curves of the probability of still being in the ED as a function of time for the strata of age category, race, sex, and ethnicity.

The p-values were computed using the log-rank test. The curves are truncated at the length of stay of 24hour for better visual presentation.

ED - emergency department 


\section{Cureus}
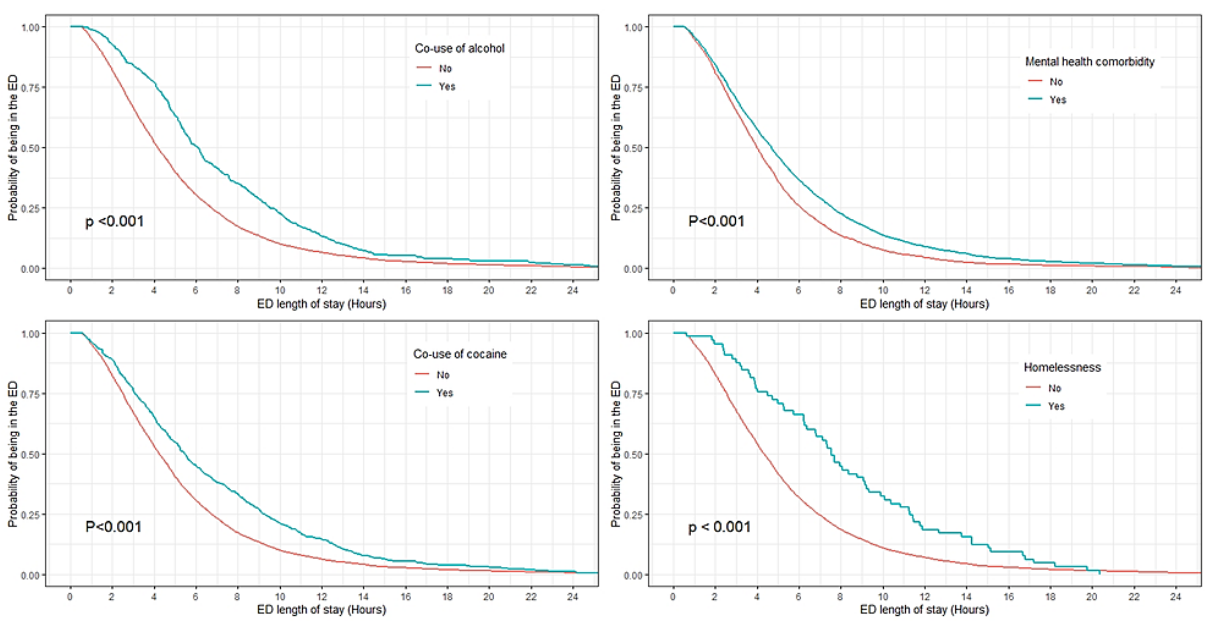

FIGURE 3: Kaplan-Meir curves of the probability of still being in the ED as a function of time for the strata of co-use of alcohol, co-use of cocaine, the presence of mental health comorbidity, and homelessness.

The $p$-values were computed using the log-rank test. The curves are truncated at the length of stay of 24hour for better visual presentation.

ED - emergency department

\section{Results from multivariable AFT models of ED LOS}

The AFT models with a Gamma distribution were found to be the best fitting models based on the goodnessof-fit test using the likelihood-ratio statistic and AIC. The results from the AFT model are presented in Table 2. The results showed that co-use of cocaine, co-use of alcohol, mental health comorbidity, homelessness, number of comorbidities, and triage index were significantly associated with the LOS of the opioid-related ED visits. Similarly, patients' demographic factors such as age, sex, race, and ethnicity were also significantly associated with the ED LOS. The patients' ED arrival shift was also significant. The visit day (weekday vs weekend) and patients' insurance type were not associated with the ED LOS.

The patients with co-use of alcohol had 31\% longer LOS compared to the patients who were not diagnosed with alcohol abuse during the visit $(\mathrm{TR}=1.31, \mathrm{p}<0.001)$. Similarly, the patients with co-use of cocaine during the visit had an $18 \%$ longer LOS compared to those without co-use of cocaine $(\mathrm{TR}=1.18, \mathrm{p}<0.001)$. The patients with mental health comorbidity had $5 \%$ longer ED LOS compared to the patients without mental health comorbidity. Homelessness was associated with a 57\% longer LOS (TR=1.57, $\mathrm{p}<0.001)$. Similarly, incremental age ( 1 year, $\mathrm{TR}=1.02, \mathrm{p}<0.001)$ female sex $(\mathrm{TR}=1.06, \mathrm{p}=0.001)$, Black/African American race $(\mathrm{TR}=1.07, \mathrm{p}<0.001)$, Hispanic or Latino ethnicity $(\mathrm{TR}=1.11, \mathrm{p}=0.048)$ were associated with an increased LOS of the ED visits. 


\section{Cureus}

\begin{tabular}{|c|c|c|}
\hline Variable & Time ratio (TR) ( $95 \% \mathrm{Cl}$ of TR) & P-value \\
\hline Age & $1.02(1.01,1.04)$ & 0.010 \\
\hline Female vs Male & $1.06(1.02,1.10)$ & 0.001 \\
\hline Black/African American vs White & $1.07(1.02,1.12)$ & 0.002 \\
\hline Hispanic or Latino vs Non-Hispanic or Latino & $1.11(1.01,1.23)$ & 0.048 \\
\hline Medicaid vs Commercial Insurance & $0.95(0.90,1.01)$ & 0.151 \\
\hline Medicare vs Commercial Insurance & $1.04(0.95,1.13)$ & 0.328 \\
\hline Weekend vs Weekday Arrival & $0.97(0.94,1.02)$ & 0.295 \\
\hline Day Shift vs Night Shift Arrival & $0.96(0.92,1.01)$ & 0.163 \\
\hline Evening Shift vs Night Shift Arrival & $1.04(0.99,1.09)$ & 0.088 \\
\hline Triage Severity Index & $0.72(0.70,0.74)$ & $<0.001$ \\
\hline Elixhauser Comorbidity Count & $1.02(1.01,1.03)$ & $<0.001$ \\
\hline Co-use of Alcohol & $1.31(1.23,1.40)$ & $<0.001$ \\
\hline Co-use of Cocaine & $1.18(1.11,1.25)$ & $<0.001$ \\
\hline Mental Health Comorbidity & $1.05(1.01,1.09)$ & 0.028 \\
\hline Homeless & $1.57(1.32,1.86)$ & $<0.001$ \\
\hline
\end{tabular}

TABLE 2: Results from the accelerated failure time (AFT) model of length of stay of opioid-related ED visits.

ED - emergency department

\section{Limitations}

This study has several limitations. The data for the study came from a single hospital system so the results may not be generalizable to other hospitals. The list of covariates evaluated to include in the models was not exclusive and there might be other possible factors associated with LOS, which were not included in the model. Previous studies have reported ED occupancy [23-25], and service processes like laboratory tests and imaging [26] to be significant predictors of LOS in ED. Also, the analysis does not control for visits related to opioid overdose and opioid withdrawal, which may have different implications on the ED LOS.

\section{Discussion}

Co-use of alcohol and co-use of cocaine were significantly associated with increased ED LOS of opioidrelated ED visits. Patients with co-use of alcohol and patients with co-use of cocaine spent $31 \%$ and $18 \%$ longer times in ED respectively compared to the patients without that co-usage. Co-use of alcohol and couse of cocaine might have increased the ED LOS due to additional health complexities related to multiple drug usage. These patients may also have to go through additional laboratory tests and procedures while in ED. To the knowledge of the author, there is no study that reports on how co-use of alcohol and co-use cocaine among patients with opioid abuse affects ED LOS and hospital utilization in general. However, past studies have reported an association of alcohol use and an increased ED LOS in mental health patients [15], minimally injured patients [27], and psychiatric patients [28]. A previous study reported that psychiatric patients with a negative toxicology screen result stayed an average of 12 hours (10.6 to 13.5), whereas patients who presented with any serum alcohol level greater than zero, with or without other substances, had average ED stays of 14.5 hours (12.3 to 17.2) [28]. These results indicate that alcohol abuse is an important factor associated with the increased LOS irrespective of patient populations.

Homelessness was associated with an increase in ED LOS with a TR of 1.57, which corresponds to a 57\% longer LOS compared to patients who were not homeless. Previous studies have reported similar results but in different patient populations. Homelessness was reported to be associated with increased LOS by $45 \%$ in psychiatric patients [29], and by $7 \%$ in patients with a history of substance use disorder (SUD) [5]. The relationship between longer ED LOS and homelessness has been implicated in higher rates of medical comorbidities and mental health disorders among homeless patients [30]. To note, the analysis in this study adjusted for a number of comorbidities, and the presence of mental health comorbidity. The longer LOS of 
homeless patients could be related to delays in the discharge of homeless patients due to a lack of immediate safe disposition and post-discharge shelters.

Sex was another factor associated with ED LOS with the females spending longer time in the ED compared to the males. The results showed a TR of 1.09 for females to males, which corresponds to a $9 \%$ longer LOS for females compared to males. Results from previous studies on the effect of sex on ED LOS are not consistent and varied by patient population. A previous study analyzing the LOS of the total ED population reported similar results with females spending significantly longer time in ED than males, but the effect size was much smaller [10]. However, in a population-based study in Ontario, Canada, sex was reported not to be a significant determinant of prolonged ED stay [13]. Further, we find a racial and ethnic disparity on the ED LOS with Black/African American, and Hispanic or Latino spending 7\% and 11\% longer time in the ED compared to their White, and Non-Hispanic or Latino counterparts. These findings support previously documented racial and ethnic disparities in timely receipt of ED care and LOS in different patient populations [31-33]. Bekmezian et. al explained similar ethnic and racial disparity in ED LOS in the pediatric population to be possibly caused by language barrier [31], and this might be a potential factor in opioidrelated visits. However, we need prospective studies, and root-cause analyses to better explain these racial and ethnic disparities.

\section{Conclusions}

This study is unique in exploring the effect of patient's characteristics on LOS of opioid-related visits. We find that female sex, Black/African American race, Hispanic or Latino ethnicity, co-use of alcohol, co-use of cocaine, homelessness and the presence of mental health comorbidity are associated with increased ED LOS. These results could be helpful to physicians and patients to better anticipate an individual's LOS and could help administrators devise tailored interventions and processes to reduce protracted ED stays.

\section{Additional Information \\ Disclosures}

Human subjects: Consent was obtained or waived by all participants in this study. Christiana Care Health System Institutional Review Board issued approval CCC\# 39082. This is to officially inform you that your protocol was approved by Expedited Review per 45 CFR 46.110(f)(5) with a Waiver of Consent 45 CFR 46.116(d) and Waiver of HIPAA Authorization 45 CFR 46.164.512(1)(i)2(ii) by Jerry Castellano, Pharm. D, CIP, Corporate Director of Christiana Care Health System Institutional Review Board (IRB00000480). Animal subjects: All authors have confirmed that this study did not involve animal subjects or tissue. Conflicts of interest: In compliance with the ICMJE uniform disclosure form, all authors declare the following: Payment/services info: All authors have declared that no financial support was received from any organization for the submitted work. Financial relationships: All authors have declared that they have no financial relationships at present or within the previous three years with any organizations that might have an interest in the submitted work. Other relationships: All authors have declared that there are no other relationships or activities that could appear to have influenced the submitted work.

\section{Acknowledgements}

The author would like to acknowledge invaluable assistance with database creation by J Thomas Laughery. The author acknowledges Claudine T. Jurkovitz and Zekarias Berhane for their guidance on data analysis and interpretation of results.

\section{References}

1. Karaca Z, Wong HS, Mutter RL: Duration of patients' visits to the hospital emergency department . BMC Emerg Med. 2012, 12:15. 10.1186/1471-227X-12-15

2. Derose SF, Gabayan GZ, Chiu VY, Yiu SC, Sun BC: Emergency department crowding predicts admission length-of-stay but not mortality in a large health system. Med Care. 2014, 52:602-11. 10.1097/MLR.0000000000000141

3. Section 3. Measuring Emergency Department Performance | Agency for Healthcare Research and Quality . (2021). Accessed: February 19, 2021: https://www.ahrq.gov/research/findings/finalreports/ptflow/section3.html.

4. Opioid Hospital Stays Emergency Department Visits - HCUP Fast Stats . (2020). Accessed: November 10, 2020: https://www.hcup-us.ahrq.gov/faststats/OpioidUseServlet.

5. Subedi K, Zhang Z, Horton T, Jurkovitz C: 3459 Modeling emergency department length of stay of patients with substance use disorder using an accelerated failure time model. J Clin Transl Sci. 2019, 3:49. $10.1017 /$ cts.2019.116

6. Fahimi J, Aurrecoechea A, Anderson E, Herring A, Alter H: Substance abuse and mental health visits among adolescents presenting to US emergency departments. Pediatr Emerg Care. 2015, 31:331-8. 10.1097/PEC.0000000000000421

7. Costs of Emergency Department Visits for Mental and Substance Use Disorders in the United States, 2017 \#257. (2021). Accessed: February 19, 2021: https://www.hcup-us.ahrq.gov/reports/statbriefs/sb257-EDCosts-Mental-Substance-Use-Disorders-2017.jsp.

8. Pitts SR, Vaughns FL, Gautreau MA, Cogdell MW, Meisel Z: A cross-sectional study of emergency 
department boarding practices in the United States. Acad Emerg Med. 2014, 21:497-503. 10.1111/acem.12375

9. Chaou CH, Chiu TF, Yen AM, Ng CJ, Chen HH: Analyzing factors affecting emergency department length of stay-using a competing risk-accelerated failure time model. Medicine (Baltimore). 2016, 95:e3263. 10.1097/MD.0000000000003263

10. Chaou CH, Chen HH, Chang SH, Tang P, Pan SL, Yen AM, Chiu TF: Predicting length of stay among patients discharged from the emergency department-using an accelerated failure time model. PLoS One. 2017, 12:e0165756. 10.1371/journal.pone.0165756

11. Wiler JL, Handel DA, Ginde AA, et al.: Predictors of patient length of stay in 9 emergency departments . Am J Emerg Med. 2012, 30:1860-4. 10.1016/j.ajem.2012.03.028

12. Davis B, Sullivan S, Levine A, Dallara J: Factors affecting ED length-of-stay in surgical critical care patients . Am J Emerg Med. 1995, 13:495-500. 10.1016/0735-6757(95)90155-8

13. Rose L, Scales DC, Atzema C, et al.: Emergency department length of stay for critical care admissions. A population-based study. Ann Am Thorac Soc. 2016, 13:1324-32. 10.1513/AnnalsATS.201511-7730C

14. Zhu JM, Singhal A, Hsia RY: Emergency department length-of-stay for psychiatric visits was significantly longer than for nonpsychiatric visits, 2002-11. Health Aff (Millwood). 2016, 35:1698-706. 10.1377/hlthaff.2016.0344

15. Pearlmutter MD, Dwyer KH, Burke LG, Rathlev N, Maranda L, Volturo G: Analysis of emergency department length of stay for mental health patients at Ten Massachusetts Emergency Departments. Ann Emerg Med. 2017, 70:193-202.e16. 10.1016/j.annemergmed.2016.10.005

16. Stephens RJ, White SE, Cudnik M, Patterson ES: Factors associated with longer length of stay for mental health emergency department patients. J Emerg Med. 2014, 47:412-9. 10.1016/j.jemermed.2014.04.040

17. Hoffmann JA, Stack AM, Monuteaux MC, Levin R, Lee LK: Factors associated with boarding and length of stay for pediatric mental health emergency visits. Am J Emerg Med. 2019, 37:1829-35. 10.1016/j.ajem.2018.12.041

18. Klein LR, Driver BE, Miner JR, Martel ML, Cole JB: Emergency department length of stay for ethanol intoxication encounters. Am J Emerg Med. 2018, 36:1209-14. 10.1016/j.ajem.2017.12.017

19. Table 4, ICD-9-CM and ICD-10-CM Diagnosis Codes Defining Opioid Use Disorder (OUD) . (2020). Accessed: April 14, 2020: https://www.ncbi.nlm.nih.gov/books/NBK557173/table/sb256.tab7/.

20. Elixhauser Comorbidity Software Refined for ICD-10-CM . (2021). Accessed: February 19, 2021: https://www.hcup-us.ahrq.gov/toolssoftware/comorbidityicd10/comorbidity_icd10.jsp.

21. Substance Use Disorders and ICD-10-CM Coding . (2021). Accessed: February 19, 2021: https://www.apaservices.org/practice/update/2015/09-10/substance-disorders.

22. Modelling Survival Data in Medical Research - David Collett. (2021). Accessed: February 19, 2021 : https://books.google.com/books.

23. Kreindler SA, Cui Y, Metge CJ, Raynard M: Patient characteristics associated with longer emergency department stay: a rapid review. Emerg Med J. 2016, 33:194-9. 10.1136/emermed-2015-204913

24. Li L, Georgiou A, Vecellio E, Eigenstetter A, Toouli G, Wilson R, Westbrook JI: The effect of laboratory testing on emergency department length of stay: a multihospital longitudinal study applying a crossclassified random-effect modeling approach. Acad Emerg Med. 2015, 22:38-46. 10.1111/acem.12565

25. Forster AJ, Stiell I, Wells G, Lee AJ, Van Walraven C: The effect of hospital occupancy on emergency department length of stay and patient disposition. Acad Emerg Med. 2003, 10:127-33. 10.1197/aemj.10.2.127

26. Yoon P, Steiner I, Reinhardt G: Analysis of factors influencing length of stay in the emergency department . Can J Emerg Med. 2003, 5:155-61. 10.1017/s1481803500006539

27. Park JH, Park JO, Ro YS, Shin SD: Effect of alcohol use on emergency department length of stay among minimally injured patients based on mechanism of injury: multicenter observational study. Clin Exp Emerg Med. 2018, 5:7-13. 10.15441/ceem.16.180

28. Weiss AP, Chang G, Rauch SL, et al.: Patient- and practice-related determinants of emergency department length of stay for patients with psychiatric illness. Ann Emerg Med. 2012, 60:162-71.e5. 10.1016/j.annemergmed.2012.01.037

29. Tulloch AD, Khondoker MR, Fearon P, David AS: Associations of homelessness and residential mobility with length of stay after acute psychiatric admission. BMC Psychiatry. 2012, 12:121. 10.1186/1471-244X-12-121

30. Russolillo A, Moniruzzaman A, Parpouchi M, Currie LB, Somers JM: A 10-year retrospective analysis of hospital admissions and length of stay among a cohort of homeless adults in Vancouver, Canada. BMC Health Serv Res. 2016, 16:60. 10.1186/s12913-016-1316-7

31. Bekmezian A, Chung PJ, Cabana MD, Maselli JH, Hilton JF, Hersh AL: Factors associated with prolonged emergency department length of stay for admitted children. Pediatr Emerg Care. 2011, 27:110-5. 10.1097/PEC.0b013e31820943e4

32. James CA, Bourgeois FT, Shannon MW: Association of race/ethnicity with emergency department wait times. Pediatrics. 2005, 115:e310-5. 10.1542/peds.2004-1541

33. Sonnenfeld N, Pitts SR, Schappert SM, Decker SL: Emergency department volume and racial and ethnic differences in waiting times in the United States. Med Care. 2012, 50:335-41.

10.1097/MLR.0b013e318245a53c 